\title{
An allosteric network in spastin couples multiple activities required for microtubule severing
}

\author{
Colby R. Sandate ${ }^{1}$, Agnieszka Szyk ${ }^{2}$, Elena Zehr ${ }^{2}$, Gabriel C. Lander ${ }^{1, \ddagger}$, Antonina Roll- \\ Mecak $2,3, \ddagger$ \\ ${ }^{1}$ The Scripps Research Institute, La Jolla, CA 92037 \\ ${ }^{2}$ Cell Biology and Biophysics Unit, Porter Neuroscience Research Center, National Institute of \\ Neurological Disorders and Stroke, Bethesda, MD 20892 \\ ${ }^{3}$ Biochemistry and Biophysics Center, National Heart, Lung and Blood Institute, Bethesda, MD \\ 20892
}

\begin{abstract}
The AAA+ ATPase spastin remodels microtubule arrays through severing, and its mutation is the most common cause of hereditary spastic paraplegias (HSP). Polyglutamylation of the tubulin Cterminal tail recruits spastin to microtubules and modulates severing activity. Here, we present a $\sim 3.2$ Å resolution cryo-EM structure of the Drosophila melanogaster spastin hexamer with a polyglutamate peptide bound in its central pore. Two electropositive loops arranged in a doublehelical staircase coordinate the substrate sidechains. The structure reveals how concurrent nucleotide and substrate binding organizes the conserved spastin pore loops into an ordered network that is allosterically coupled to oligomerization, and suggests how tubulin tail engagement activates spastin for microtubule disassembly. This allosteric coupling may apply generally in organizing AAA+ protein translocases into their active conformations. We show that this allosteric network is essential for severing and is a hotspot for HSP mutations.
\end{abstract}

Spastin is a microtubule-severing AAA ATPase (ATPases Associated with diverse cellular Activities) whose function is important in basic cell biological processes ranging from neurogenesis and axonal maintenance to nuclear envelope breakdown, vesicle trafficking, mitosis, and cytokinesis ${ }^{1}$. Spastin is recruited to endosomes and the midbody through its interaction with ESCRTIII and is thought to participate in the coordinated remodeling of membranes and microtubules ${ }^{1}$. Spastin ATPase activity is stimulated by microtubules ${ }^{2-4}$ and microtubule severing activity requires adenosine triphosphate (ATP) hydrolysis ${ }^{2,5-7}$. Spastin

\footnotetext{
Users may view, print, copy, and download text and data-mine the content in such documents, for the purposes of academic research, subject always to the full Conditions of use:http://www.nature.com/authors/editorial_policies/license.html\#terms

${ }^{\ddagger}$ Co-corresponding Authors: Gabriel C. Lander, Department of Integrative Structural and Computational Biology, The Scripps Research Institute, HZ 175, 10550 N. Torrey Pines Rd., La Jolla, CA 92037, Phone: (858) 784-8793, glander@ scripps.edu; Antonina Roll-Mecak, Cell Biology and Biophysics Unit, Porter Neuroscience Research Center, National Institutes of Health, Building 35, Room 3B-203, 35 Convent Drive, MSC 3700, Bethesda, MD 20892-3700, Phone: 301-814-8119, antonina @ mail.nih.gov. Author Contributions

C.R.S. froze EM grids, collected and processed EM data and built atomic models. A.S. purified all protein, performed AUC and ATPase assays. E.A.Z. performed severing assays. C.R.S., G.C.L. and A.R.M. interpreted structural model and wrote manuscript.

Competing financial interests

The authors declare no competing financial interests.
} 
mutations are responsible for $\sim 50 \%$ of hereditary spastic paraplegias (HSPs), a large and clinically diverse family of neurodegenerative disorders ${ }^{8}$. In the pure form of the disease, HSP patients typically exhibit axonal degeneration in motor axons of the corticospinal tract, leading to progressive lower limb spasticity and weakness ${ }^{8-10}$. Studies in model organisms ${ }^{11}$ as well as patient derived induced pluripotent stem cells ${ }^{12}$ have shown that neurons are especially sensitive to spastin gene dosage. Cellular and biochemical studies have shown that many of the disease associated mutations examined impair microtubule severing and ATP hydrolysis ${ }^{5-7,13}$. However, the etiology of spastin induced HSP is still poorly understood.

Spastin has a modular structure comprising a three-helix bundle microtubule-interacting and trafficking (MIT) domain, a poorly-conserved linker region, and an AAA+ ATPase domain. This domain architecture is shared among all three known microtubule severing enzymes spastin, katanin, and fidgetin ${ }^{1}$. The ATPase domain is structurally homologous to that of other members of the AAA+ protein family and consists of an $\alpha / \beta$ nucleotide-binding domain (NBD) and a four-helix bundle domain (HBD). Both spastin and katanin differ from other AAA+ ATPase enzymes in that they contain two additional helices within the NBD that are essential for severing 6,14 . The AAA+ ATPase domains hexamerize in a nucleotidedependent manner and the tubulin substrate lowers the critical concentration for oligomerization ${ }^{15,16}$. Consistent with this, high-affinity binding of spastin to microtubules is highly cooperative and requires ATP 4 . The substrate-dependent oligomerization is likely important for the specificity and timing of action of these AAA+ ATPases in the cell, as unregulated spastin and katanin microtubule-severing activity is highly deleterious ${ }^{17-19}$. The majority of disease associated mutations of the SPAST gene are found in the ATPase domain of spastin.

Microtubule severing by spastin requires the $\beta$-tubulin C-terminal tail, an intrinsically disordered element that decorates the microtubule surface. While the a-tubulin tail contributes to binding, it is not required for severing activity ${ }^{20}$. It was proposed that microtubule severing involves the hexamerization of spastin protomers around the $\sim 20$ residue, negatively charged tubulin tail, and the subsequent pulling of the tubulin subunit out the microtubule lattice using ATP-driven conformational changes in three pore loops that help translocate the substrate through the central pore of the hexameric ATPase ${ }^{6,21}$. However, there has been no direct demonstration of the tubulin tail engagement by the spastin pore nor any atomistic structural information on the spastin hexamer or its interaction with the tubulin tail substrate. Spastin's interaction with the microtubule is strongly enhanced by polyglutamylation of the $\beta$-tubulin tail ${ }^{20}$, a post-translational modification that involves the addition of multiple glutamate chains of variable lengths; unmodified microtubules are not effectively severed at in vivo spastin concentrations ${ }^{20,22}$. As many as 21 glutamates have been detected on tubulin tails in vivo ${ }^{23,24}$. Polyglutamylation is highly abundant in neurons where spastin activity is required for neurite extension as well as axonal maintenance and regeneration ${ }^{11}$.

Here, we present the cryo-EM structure of the spastin hexamer in complex with a polyglutamate peptide at $\sim 3.2 \AA$ resolution. The structure reveals an asymmetric hexamer with the AAA domains arranged in a split lock-washer conformation. Two conserved pore 
loops from the six protomers form a double-helical staircase gripping the odd and even glutamates of the substrate peptide, respectively. Residues in this double helix are positively charged and neutralize the electronegative polyglutamate peptide, consistent with the regulation of microtubule severing by polyglutamylation of tubulin tails. The substrate binding pore loops are allosterically coupled to the ATP binding site as well as the oligomerization interfaces, providing a structural explanation for the tubulin substrate binding-induced spastin activation. The majority of the residues in this allosteric network are mutated in HSP patients, underscoring their importance to spastin function. Our comprehensive structural analysis of all reported HSP-associated spastin missense mutations in its AAA core provides a framework for understanding spastin molecular dysfunction.

\section{RESULTS}

\section{Spastin forms a hexameric spiral}

Unlike many AAA+ ATPases, spastin exists as a monomer or dimer in the absence of nucleotide or bound to ADP, and only assembles into hexamers upon ATP-binding ${ }^{3,6}$ This hexamer is labile and falls apart at lower concentrations ${ }^{6}$. In order to stabilize the hexameric state for structural studies by cryo-electron microscopy (cryo-EM) we used a commonly used mutation in the Walker B motif of the ATPase domain (E583Q), which retains ATP binding but prevents ATP hydrolysis ${ }^{6,13}$. Analytical ultracentrifugation (AUC) shows that this construct assembles into hexamers in the presence of ATP at concentrations as low as 6 $\mu \mathrm{M}$ (Supplementary Fig. 1a). Initial structure determination yielded a reconstruction of the spastin hexamer limited to $\sim 3.8 \AA$ resolution showing cryo-EM density of adventitious peptide binding. As polyglutamylation enhances substrate binding ${ }^{20}$ and spastin has been shown to interact with polyglutamate peptides, we incubated our spastin preparation with polyglutamate (Methods). This increased the proportion of intact hexamers and allowed structural determination of the spastin-peptide complex to $\sim 3.2 \AA$ resolution (Table 1, Fig. 1, Supplementary Figs. 2 and 3). Poly-glutamate activates spastin ATPase with a maximal activation of $\sim$ six-fold above the basal level (Supplementary Fig. 1b), comparable to the activation reported with microtubules ${ }^{2}$. Analytical centrifugation with a fluorescently labeled poly-glutamate peptide shows that it comigrates with the spastin hexamer (Supplementary Fig. 1c). The choice of polyglutamate also overcomes the experimental issue of placing enzymes in a specific register with respect to the tubulin tail sequence which is overrepresented in polyglutamates, but also contains other amino acid residues.

The overall architecture of the spastin hexamer resembles that of a split lock-washer, as previously observed for other homohexameric ATPases ${ }^{25,26}$, including the related severing enzyme katanin ${ }^{14}$ (Fig. 1a). The individual protomers assemble into a right-handed spiral, with a $\sim 6 \AA$ rise for each protomer and a $\sim 35 \AA$ gap between the topmost and lowest protomers. The large (NBD) subdomain of the ATPase forms the central pore of the hexamer, with the HBD directed peripherally and in contact with the NBD of the neighboring protomer. The cryo-EM density corresponding to the "gating" protomers A and $\mathrm{F}$ is lower in resolution (particularly the HBD of protomer F) than that of the central four protomers, indicative of increased flexibility (Supplementary Fig. 3b). The MIT (which is dispensable for microtubule severing activity ${ }^{13}$ ) and most of the linker are not observed in 
the reconstruction, and were likely averaged out during image processing due to their conformational variability, as seen for katanin ${ }^{14}$. However, the EM map was of sufficient quality to model $98.7 \%$ of the residues in the hexameric ATPase domains (Table 1; Fig. 1b, Supplementary Fig. 4a).

Our reconstruction contains interpretable cryo-EM density features within each of the six nucleotide binding pockets. With the exception of the gating protomer $\mathrm{F}$, all protomers contain a clearly defined ATP molecule with a magnesium ion coordinated between the $\beta$ and $\gamma$-phosphates and T530 of the phosphate binding loop (Supplementary Fig. 4b). R640 and R641 from the neighboring protomer complete the ATP binding site for all protomers except for the gating protomer F. The Arginine finger residue R641 is in a catalytically competent conformation and within $\mathrm{H}$-bonding distance from the $\gamma$-phosphate (Supplementary Fig. 4b). The nucleotide binding pocket of protomer F is not as well resolved as in the other protomers (Supplementary Fig. 4b), and the cryo-EM density could correspond to either ADP or ATP. The binding pocket of protomer F is exposed to solution, presumably flexible, and the lack of clear density for the $\gamma$-phosphate could arise from either incomplete coordination of the ATP or to background hydrolysis of the ATP. However, comparison of the overall fold of this F protomer to the ATP-bound protomers (Supplementary Fig. 5a) reveals that their structures are highly similar $(0.695 \AA \mathrm{Ca}$ RMSD). In contrast, comparing the structures of the apo spastin monomer (PDB 3B9P) ${ }^{6}$ with the ATP-bound protomers in our structure (Supplementary Fig. 5b) shows extensive conformational rearrangement of the NBD, as well as a $\sim 9^{\circ}$ rotation of the HBD relative to the NBD, consistent with nucleotide binding being the main driver of the conformational changes required for hexamerization of the ATPase protomers. Notably, three conserved and functionally important loops in the AAA domain are disordered or poorly ordered in the nucleotide-free structures of spastin 6,27 , but are well-ordered in our hexamer structure and line the central pore, where they are involved in substrate binding and inter-protomer contacts. Linker residues 455-463 preceding a 1 also become ordered in the hexamer and are stabilized through interactions with helix a 1 from the neighboring protomer (Supplementary Fig. 6a). Interestingly, the structure of this linker region is distinct from the "fishhook" motif found in katanin, which decorates the $\mathrm{N}$-terminal side of the central pore and also participates in protomer-protomer contacts ${ }^{14}$. Helix a 1 is unique to severing enzymes, and mutations in this helix impair severing ${ }^{6}$. The linker connecting helices a 1 and a 2 moves in concert with the closure of the NBD and HBD around the nucleotide, establishing stabilizing interactions with the $a 1-\beta 4$ loop of the neighboring lower protomer. The latter loop is disordered in the apo structure ${ }^{6}$. On the opposite face of the hexamer, the C-terminus of the AAA domain (res. 753-757), which is disordered in prior crystal structures ${ }^{6,27}$, is engaged in intimate contacts with the a 10- a 11 loop of the neighboring protomer (Supplementary Fig. 6b), and together with helix a 11 forms a belt around the hexamer as in katanin ${ }^{14}$. Invariant Y753 at the end of helix a 11 is part of the interface with the a11- a 12 linker of the neighboring protomer (Supplementary Fig. 6c), and its mutation severely impairs severing ${ }^{6}$. 


\section{A double-helical staircase of pore loops coordinates the substrate}

Density for the polyglutamate peptide is clearly visible at the center of the spastin hexamer (Fig. 2). While the added polyglutamate peptide ranges in length from 10 to 37 amino acids, only the 15 residues that undergo stabilizing interactions with the spastin AAA core are clearly visible (Fig. 2a, b and Fig. 3a). We were unable to assign the polarity of the substrate peptide in the cryo-EM density with certainty at this resolution, as both polarities resulted in acceptable fits to the map (Supplementary Fig. 7) and neither orientation resulted in an increase in stabilizing interactions with the spastin hexamer. We modeled the polarity of the peptide with the $\mathrm{N}$-terminus of the peptide engaged to the uppermost protomer (protomer A), and the C-terminus closest to the lowest protomer (protomer F). We note that structural and functional studies have so far been unable to unambiguously assign the polarity of the substrate peptide for the meiotic class of AAA ATPases to which spastin and katanin belong, and the current assigned polarity has been inferred from functional studies and analogy with other AAA+ ATPases, including VPS4 ${ }^{28}$, $\mathrm{HSP} 104^{29}$ and the proteasome ${ }^{30}$. Interestingly, a recent study of the AAA ATPase VAT shows that it is able to translocate peptides in either direction $^{31}$. It is possible that the polarity of the peptide through the spastin pore is not dictated by pore interactions alone and also results from the engagement of other interfaces with the microtubule substrate.

Consistent with the cryo-EM structures of other AAA+ ATPases, pore loop 1 (555-562), which is characterized as having a conserved aromatic residue important for substrate translocation $^{32}$, interacts directly with the bound substrate (Fig. 2b and Figs. 3a,

b) $28,30,33-35$. Conserved Y556 intercalates between $n$ and $n+2$ residues of the substrate, sandwiching the glutamate sidechain and forming a hydrogen bond with one of the carboxyl oxygens (Fig. 3a, b). Consistent with its role in substrate engagement, its mutation abolishes severing while retaining full ATPase activity (Fig. 4). This intercalating organization between pore loops and substrate has been previously observed in other ATPases ${ }^{28,29,33}$ and likely serves as a general mode of substrate interaction for AAA+ ATPases.

Together with invariant K555, which forms a salt bridge with the glutamate of the $n+1$ substrate residue, Y556 constitutes the first of two pore-loop spiral staircases that coordinate the substrate. The sidechain of pore loop 1 residue K555 is also likely stabilized by a hydrogen bond with conserved E462 in helix a 1, the secondary structure element unique to severing enzymes ${ }^{6,14}$. VPS4, the other member of the meiotic clade of AAA ATPases, has a short $\beta$-strand at this location ${ }^{36}$. Mutation of residues in a 1 impair severing without a notable effect on ATPase activity ${ }^{6}$. Invariant H596 and R601 of pore loop 2 (594-601) form electrostatic interactions with every other peptide sidechain substrate $(n, n+2)$, generating a second spiral staircase (Fig. 2b and Fig. 3b). Mutation of either of these residues inactivates severing and impairs ATPase activity ${ }^{6}$. Together, pore loops 1 and 2 enclose the poly-Glu substrate with a double helix of positively charged residues (Fig. 2b and Fig. 3b), neutralizing the highly negative charge of the peptide and providing substrate specificity for either the poly-Glu chains on the tubulin tails, or the $\beta$-tubulin tails themselves, which contain a high density of negatively charged residues (8-10 glutamates in addition to $2-3$ aspartates) ${ }^{37}$. This double coordination by both pore loop 1 and 2 is distinct from VPS4, whose pore loop 2 does not directly contact its polypeptide substrate ${ }^{28}$. Participation of pore 
loop 2 in substrate translocation has been previously inferred from crosslinking studies of $\mathrm{ClpA}^{38}$, where mutagenesis of certain residues within the loop inhibits substrate degradation but not substrate binding. Additionally, crystallographic studies of HSP104 show pore loop 2 interacting with an adjacent N' domain molecule, acting as a "substrate mimic" 39 .

\section{An allosteric network mutated in HSP couples substrate binding to oligomerization}

A third highly conserved pore loop in the AAA domain (629-637) runs nearly orthogonal to pore loop 2, and does not contact the substrate peptide directly, but functions as a structural bridge linking the substrate-interacting pore loops to the nucleotide-binding pocket (Fig. 3c). Invariant E561 in a4, which immediately follows pore loop 1, is within proximity of $\mathrm{H}$ bonding interactions, with invariant R601 from pore loop 2 of the same protomer, potentially allowing direct coordination between these two pore loops. Pore loop 2 is disordered in crystal structures of the apo spastin monomer ${ }^{6,27}$ (Supplementary Fig. 5b), and the fact that we observe direct interactions between R601 and substrate strongly suggests that the E561R601 interaction depends on the presence of substrate. Consistent with this role, mutation of E561 abolishes severing, while still retaining ATPase activity (Fig. 4b,c). R601 is also within H-bonding distance to S599 of the lower neighboring protomer, thus possibly linking substrate binding to oligomerization (Supplementary Fig. 6d). Both S599 and R601 are mutated in HSP (Supplementary Table 1) and the R601L disease mutation impairs ATPase and abolishes microtubule severing ${ }^{6}$. The organization of these pore loops gives rise to an interaction network wherein invariant R600 of pore loop 2 forms a salt bridge with invariant E633 in pore loop 3 of the next, lower protomer, which in turn interacts with invariant R630 which H-bonds with the backbone of R591 in loop 2 of the upper protomer (Fig. 3c). Invariant $\mathrm{N} 629$ in pore loop 3 is within H-bonding distance from the $\gamma$-phosphate of the ATP of the same protomer and thus can potentially contribute to nucleotide sensing in addition to the traditional sensor residues. Consistent with this, mutation of N629 abolishes both ATPase activity and severing (Fig. 4b,c). Together with invariant D585, N629 is also within H-bonding distance of invariant R591 of the neighboring lower protomer, which in turn is within hydrogen bonding distance to the catalytic glutamate in the Walker B motif (E583) of the higher protomer (Fig. 5a). The R591 backbone is also stabilized through interaction with the R630 sidechain from the lower protomer (Figs. 5b). Thus, R591 is at the center of an allosteric network that can couple the substrate binding loops to ATP hydrolysis and oligomerization. Moreover, the conformation of pore loop 3 containing R630 is coupled through a network of hydrophobic residues to helix a 11 involved in oligomerization interactions between HBD domains (Fig. 5b and Supplementary Fig. 6b). Mutation of Y753 at this oligomerization interface severely impairs severing ${ }^{6}$. Strikingly, all residues in this extensive network are mutated in HSP patients, highlighting their importance for spastin function (Fig. 5 and Supplementary Table 1). Thus, our structure sheds light on how ATP and peptide substrate binding establish a network of intra- and inter-protomer interactions that stabilize the spastin hexamer. Since spastin intracellular concentrations are below the critical concentration for hexamer assembly ${ }^{6,22}$, we speculate that this pore loop network primes spastin for assembly around the tubulin tails for effective microtubule severing. 


\section{Effect of HSP mutations on spastin activity}

There are over $200 \mathrm{HSP}$-associated spastin mutations reported to date and 30\% of them are missense mutations ${ }^{10}$. The majority of spastin missense mutations linked to HSP are located within the ATPase domain ${ }^{10}$, and several missense disease mutations examined have been shown to inactivate or severely impair microtubule severing activity in biochemical and cellular studies ${ }^{5-7,10,13,40}$. Our structure provides a mechanistic understanding of how HSP mutations in the ATPase domain lead to loss of spastin function. Based on the high sequence identity and known structural conservation between drosophila and human spastin ${ }^{6,27}$, we generated a homology model of the human spastin hexamer (Fig. 6a) that provides complete coverage of the highly-conserved AAA domain (amino acids 89-388). Structural analysis of all known missense spastin mutations identified to date in the AAA core ${ }^{6,41-61}$ suggests that most impair oligomerization, nucleotide binding, or disrupt the folding or stability of the AAA domain's structure to variable degrees (Supplementary Table 1). Several mutations map directly to the nucleotide binding pocket (Fig. 6b) and impair nucleotide binding (I344K, G385W, N386K, K388R, M390V, R498S) and thus hexamerization. One mutation interferes with magnesium coordination (D441G). Two disease mutations affect ATP hydrolysis and sensing of the $\gamma$-phosphate (E442A or K, and R499C). E442 is part of the Walker B motif and participates in catalysis in all AAA ATPases, while R499 serves as the Arg finger. The Arg finger coordinates the $\gamma$-phosphate and electrostatically stabilizes the transition state after ATP hydrolysis. N487D (N629 in our Drosophila cryo-EM structure) is likely involved in both sensing the $\gamma$-phosphate and oligomerization interactions (Figs. $6 \mathrm{~b}$ and 5a)

Other HSP mutations are likely deleterious because they disrupt the overall fold and stability of the AAA domain structure (Fig. 6c) (I406V, V423L, L426V, F427C, A428P, L447V, S458R, and L461P among others). A large number of disease mutations map to protomerprotomer interfaces and likely interfere with oligomerization (Fig. 6d) (E356G, L360P, P361L/S, R364M, L367S, F368L/S, G370R, R372G, C448Y, A551P/Y, D555G/N, E356V, G559D, I561G, R562Q, D613H, T615I). Consistent with this, cellular studies have shown that the $\mathrm{C} 448 \mathrm{Y}$ mutant is unable to associate with wild-type spastin and inhibit microtubule severing ${ }^{62}$. Thus, the preponderance of HSP mutations likely impact oligomerization interfaces or the overall stability of spastin and may lead to spastin haploinsufficiency. However, there is a large phenotypic variability in spastin-linked HSP and more recent work has shown that some spastin disease mutations can also have a toxic gain-of-function that is further exacerbated by spastin haploinsufficiency ${ }^{63}$. We note that spastin mutants that are deficient in oligomerization could still bind microtubules through a stretch of residues in the linker that connects the AAA and MIT ${ }^{3,6,7,13,64}$ domains and thus have a toxic gain of function on the microtubule cytoskeleton. Interestingly, mutations in the Arg finger R499 have been associated with early onset $\mathrm{HSP}^{41,52,53,59,65-68}$, possibly suggesting a dominant negative effect of this mutation. The Walker B residue E442 is the other strong candidate for a dominant negative mechanism, however not enough phenotypic data are currently available for this HSP mutation. Our structure will serve as a platform for the analysis of HSP mutations and the correlation between genotype and molecular phenotype. 


\section{Discussion}

Recent cryo-EM studies of AAA+ ATPase protein translocases have shown how a conserved mechanistic infrastructure can be generally utilized for effective nucleotide-driven protein translocation, while also highlighting the allosteric diversity of this family of ATPases. Even within the meiotic clade of AAA+ ATPases, we observe differences in structural motifs such as the substrate-interacting pore loops. For example, while spastin surrounds its substrate through a network of interactions that utilizes both pore-loops, the closely related family member VPS4 primarily interacts with the translocating substrate via an aromatic-containing pore loop 1 , without direct interactions from pore loop 2 . Such differences may evolve to accommodate substrate specificity or to modulate the force exerted upon a targeted polypeptide.

Given its structural homology to the C-terminal AAA domain of spastin, it is possible that the katanin ATPase establishes a nucleotide-substrate interaction network that operates under a homologous allosteric mechanism. However, in contrast to previous structural studies of katanin, we only observed the split locker-washer conformation of spastin, and were unable to identify a closed-ring conformer within our datasets as has been observed for other AAA ATPases, including the closely related meiotic clade members katanin ${ }^{14}$ and VPS $4^{28}$. One possible explanation for this could be a higher ATP-binding affinity for spastin versus katanin, which could prevent the dissociation of nucleotide in protomer $\mathrm{F}$ under our experimental conditions, a requirement for adoption of the closed-ring conformation previously observed for katanin ${ }^{14}$ and VPS4 ${ }^{28}$. We speculate that the split locker-washer conformation of spastin observed here represents a pre-hydrolysis state where all six protomers are engaged with the substrate peptide.

In summary, our structure of spastin elucidates for the first time the details of substrate engagement by a microtubule severing enzyme, and reveals how three conserved pore loops in spastin establish a conduit that links substrate binding with oligomerization and ATP hydrolysis. Our comprehensive analysis of human HSP mutations reveals the extensive coverage of disease mutations on the spastin hexamer and how they would impact all major facets of hexamer function: ATP binding, hydrolysis, and oligomerization.

\section{Online Methods}

\section{Protein expression and purification}

Full-length Drosophila melanogaster spastin ${ }^{6}$ with a Walker B mutation (E583Q) was expressed in Escherichia coli BL21DE3 cells as a N-terminal glutathione S-transferase (GST) fusion and purified by affinity chromatography, followed by Prescission protease cleavage of the GST ${ }^{69}$. After cleavage with Prescission protease, spastin was further purified on a HiTrapQ chromatography column (subtractive step) followed by a MonoS column (GE Healthcare). Spastin eluted as two peaks that were collected separately. The purer spastin peak was concentrated to $\sim 5 \mathrm{mg} / \mathrm{ml}$ and injected on a Superdex 200 size exclusion column (GE Healthcare) in $20 \mathrm{mM}$ HEPES pH 7.5, $300 \mathrm{mM} \mathrm{KCl}, 10 \mathrm{mM} \mathrm{MgCl}_{2}, 5 \mathrm{mM}$ DTT, $5 \%$ glycerol. The sample was concentrated to $10 \mathrm{mg} / \mathrm{ml}$, flash-frozen in liquid nitrogen and stored at $-80^{\circ} \mathrm{C}$ after supplementing the glycerol concentration to $15 \%$. For ATPase and 
severing assays, the gel filtration step was omitted and the protein was concentrated to $5 \mathrm{mg} / \mathrm{ml}$ and buffer exchanged into $20 \mathrm{mM}$ HEPES pH 7.5, $300 \mathrm{mM} \mathrm{KCl,} 10 \mathrm{mM} \mathrm{MgCl}_{2}, 5$ mM DTT, $15 \%$ glycerol. Single use aliquots were flash frozen in liquid nitrogen and stored at $-80^{\circ} \mathrm{C}$. All structure-based point mutants were generated using Quickchange mutagenesis and subjected to the same purification protocol as the wild-type protein.

\section{Analytical ultracentrifugation}

AUC experiments were conducted in a ProteomeLab XL-I analytical ultracentrifuge (Beckman Coulter) using absorption optics. The spastin Walker B mutant was subjected to size exclusion chromatography in $20 \mathrm{mM}$ HEPES, $300 \mathrm{mM} \mathrm{KCl}, 10 \mathrm{mM} \mathrm{MgCl} 2$ and $1 \mathrm{mM}$ TCEP. The eluted protein was used for preparation of AUC samples in the same buffer supplemented with $0.1 \mathrm{mM}$ ATP. The sample was loaded into a $12 \mathrm{~mm}$ cell, placed in a ANTi rotor and equilibrated thermally in the ultracentrifuge. After thermal equilibrium was reached at rest at $10^{\circ} \mathrm{C}$, the rotor was accelerated to $45,000 \mathrm{rpm}$ and intensity scans at 280 $\mathrm{nm}$ were started immediately and collected until no further sedimentation boundary movement was observed. Data were analyzed in terms of continuous $c(s)$ distributions using the SEDFIT program ${ }^{70}$. All accepted fits had the Root Mean Square Deviations (RMSD) less than 0.008. Partial specific volume and buffer parameters were calculated using the Sednterp (http://sednterp.unh.edu/). Sedimentation coefficients distributions were corrected to standard conditions at $20^{\circ} \mathrm{C}$ in water, $s_{20, w}$. For the detection of spastin and peptide cosedimentation, a VGSEEEEEEEEEE peptide was synthesized and purified by Biosynthesis and then labeled with Atto488 on its N-terminus using Atto488-NHS ester (Sigma- Aldrich \#41698) according the manufacturer's protocol. After labeling, peptides were RP-HPLC purified. Experiments were performed for peptide $(16.7 \mu \mathrm{M})$ alone and spastin $(10 \mu \mathrm{M}$ for the monomer or $1.67 \mu \mathrm{M}$ for the hexamer) and excess peptide $(16.7 \mu \mathrm{M})$. The samples were placed in the four-hole AN-Ti rotor and equilibrated thermally in the ultracentrifuge. After thermal equilibrium was reached at rest at $10^{\circ} \mathrm{C}$, rotor was accelerated to $50,000 \mathrm{rpm}$ and the intensity scans at $280 \mathrm{~nm}$ and $483 \mathrm{nM}$ were started immediately and collected until no further sedimentation boundary movement was observed. Data were analyzed in terms of continuous $c(s)$ distributions using the SEDFIT program. All accepted fits had the Root Mean Square Deviations (RMSD) less than 0.008. Partial specific volume and buffer parameters were calculated using the Sednterp (http://sednterp.unh.edu/). Sedimentation coefficients distributions were corrected to standard conditions at $20^{\circ} \mathrm{C}$ in water, $s_{20, \mathrm{w}}$. Modeling the 9.9s peak as a monodisperse species gives statistically identical solutions, indicating that the $9.9 \mathrm{~s}$ peak is not a mixture of oligomers ${ }^{71}$.

\section{Sample preparation for electron microscopy}

$1.2 \mathrm{mg} \mathrm{ml}^{-1}$ of spastin ${ }^{\mathrm{E} 583 \mathrm{Q}}$ was incubated on ice for $20 \mathrm{~min}$ in $20 \mathrm{mM} \mathrm{HEPEs}$ buffer (pH 7.5) containing $300 \mathrm{mM} \mathrm{KCl}, 10 \mathrm{mM} \mathrm{MgCl}_{2}, 5 \mathrm{mM}$ DTT, $1 \mathrm{mM}$ ATP and $0.05 \%$ LMNG

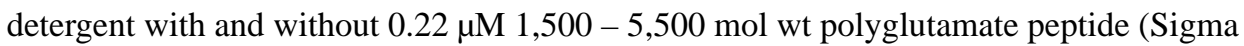
Aldrich). 2.5 $\mu$ l of sample was applied to a UltraAuFoil R1.2/1.3 300-mesh grid (Electron Microscopy Services) freshly plasma-cleaned using a Gatan Solarus (75\% argon/2\% oxygen atmosphere, $15 \mathrm{~W}$ for $7 \mathrm{~s}$ ). Grids were manually blotted with filter paper (Whatman No.1) for $\sim 4 \mathrm{~s}$ in $\mathrm{a}^{\circ} \mathrm{C}$ cold room prior to plunge freezing in liquid ethane cooled by liquid nitrogen. 


\section{Electron microscopy data acquisition}

All cryo-EM data were acquired using the Leginon automated data-acquisition program ${ }^{72}$. All image pre-processing (frame alignment, CTF estimation, particle picking) were performed in real-time using the Appion image-processing pipeline during data collection $^{73}$.Images were collected on a Thermo Fischer Talos Arctica operating at $200 \mathrm{keV}$, equipped with a Gatan K2 Summit DED, at a nominal magnification of 36,000x corresponding to a physical pixel size of $1.15 \AA$ /pixel. 2,534 movies (48 frames/movie) were collected using an exposure time of $12 \mathrm{~s}$ with an exposure rate of $5.6 \mathrm{e}^{-/} \mathrm{pixel} / \mathrm{s}$, resulting in a total dose of $52 \mathrm{e}^{-} / \AA^{2}\left(1.08 \mathrm{e}^{-} /\right.$frame $)$with a nominal defocus range from -1.0 to $-2.0 \mu \mathrm{m}$.

\section{Image processing}

A total of 564 images were used for automated particle picking using Difference of Gaussians (DoG) picker to yield 92,387 particles $^{73}$. CTFFIND4 was used for CTF estimation prior to extracting particles. Particles were Fourier-binned $4 \times 4$ and subjected to reference-free 2D classification using multivariate statistical analysis (MSA) and multireference alignment (MRA) in the Appion pipeline. The best 3 classes representing end-on, tilted and side views were selected for template-based particle picking using FindEM resulting in 2,736,856 particle picks. The $\sim 2.7$ million particles picks were extracted unbinned, and subjected to two rounds of reference-free 2D classification in cryoSPARC ${ }^{74}$ to remove non-particles and poorly aligning particles in the data (Supplementary Fig. 3). Particles from exemplary classes (strong secondary structural features present in the 2D classes) comprised a subset of 1,259,553 particles. This subset then went on to referencefree ab initio model generation in cryoSPARC, resulting in an initial 3D model. Homogeneous refinement resulted in a map with a reported resolution of $\sim 3.4 \AA$. Some disordered density above the topmost protomer was observed, which we attributed to an offregister misalignment of a subset of particles relative to the majority register. 3D classification without alignment in RELION ${ }^{75}$ using a tau-fudge value of 20 was successful in identifying the misaligned hexamers, which constituted $12 \%$ of the input particles. One class, which comprised $22 \%$ of the input particles, did not display the previously observed disordered density and was better ordered compared to the other 5 classes. 3D autorefinement in Relion with limited local and global search angles resulted in a $\sim 4.2 \AA$ map. We then used this map as a reference map for a heterogeneous refinement into 2 classes in cryoSPARC, using the original 1,259,553 particles that were kept after 2D classification. One class was significantly better ordered, and was selected for an additional round of heterogenous refinement with 2 classes. One class was selected, based on visual inspection for quality of the reconstruction, for homogenous refinement resulting in a sharpened map of $\sim 3.2 \AA$ from 488,385 particles. This final reconstruction includes only $18 \%$ of the total particles initially selected from the micrographs. We attribute this to false particle-picks, damaged particles from sample freezing, inaccurate defocus values, nonsubstrate-bound particles, and sample heterogeneity. Notably, throughout the image processing of spastin, we did not observe any evidence of an additional conformational state other than the split lock-washer conformation, even at low resolution.

An additional data set of the spastin hexamer without the addition of the polyglutamate substrate resulted in a $\sim 3.8 \AA$ A reconstruction with an unknown density within the central 
pore that resembled an unfolded polypeptide substrate (Supplementary Fig. 2). Similar densities have been observed for numerous AAA+ ATPase protein translocases ${ }^{25,28,33}$. Notably, of the $\sim 300,000$ particles selected from the images, only $\sim 30,000$ particles contributed to this final reconstruction. We were unable to identify the peptide species through mass spectrometry analysis due to its low abundance. We speculate that the unknown density is either due to a single low-abundant peptide species, or an average of many low-abundant peptides of differing sequences bound to spastin hexamers. Due to the presence of substrate density in the central pore of the hexamer, we speculated that substrate binding may have a stabilizing effect on the overall organization of the complex, and that a small minority of selected particles contained residual substrate that may have been retained through the protein purification. Indeed, pre-incubation our spastin with polyglutamate peptide, known to bind to spastin and inhibit microtubule severing ${ }^{20}$, increased the percentage of well-ordered hexamers observed in our dataset by $\sim 2$-fold and resulted in a substantial improvement in resolution to $\sim 3.2 \AA$ of the substrate-bound spastin hexamer, which allowed atomic model building (Table 1 and above).

\section{Atomic model building and refinement}

A crystal structure of the Drosophila melanogaster spastin monomer was used as a starting point $^{6}$ (PDB 3B9P) for modeling. Using Chimera ${ }^{76}$, all loops and coils were deleted, and secondary structural elements were docked into the EM map. After one round of real-space refinement in Phenix ${ }^{77}$, $\operatorname{Coot}^{78}$ was used to rebuild coils and improve main chain and side chains. The rebuilt model was then used as the initial input for a multi-model-generating pipeline ${ }^{79}$, which allowed for accessing model quality. 200 models were generated in Rosetta using the refined map and model, and the top 10 scoring models were selected for further model refinement using Phenix, using a per-residue Ca-RMSD. Regions with poor model convergence were remodeled and refined. The Molprobity ${ }^{80}$ server (http:// molprobity.biochem.duke.edu/) and PDB validation Service server (https://validatercsb-1.wwpdb.org/) were used to identify problem regions for subsequent correction in Coot. Residues 502-516 and 613-620 of protomer A are missing in the final model. The polyglutamate peptide was built de novo in Coot.

\section{ATP hydrolysis assays}

Basal ATP hydrolysis rates were measured at room temperature using the EnzChec Phosphate Assay Kit (Thermo Fisher Scientific) in $50 \mathrm{mM}$ PIPES pH 8.8, $50 \mathrm{mM} \mathrm{KCl}, 1$ $\mathrm{mM}$ EGTA, $2 \mathrm{mM} \mathrm{MgCl} 2$ and $1 \mathrm{mM}$ DTT for $50 \mathrm{nM}$ spastin and point mutants. Initial rates were calculated from the linear portion of the reaction curve after addition of $2 \mathrm{mM}$ ATP. ATPase rates were corrected by subtraction of the measured release of phosphate in the absence of ATP. Poly-glutamate stimulated ATP hydrolysis rates were measured at room temperature in $20 \mathrm{mM}$ HEPES pH 7.5, $50 \mathrm{mM} \mathrm{KCl}, 10 \mathrm{mM} \mathrm{MgCl}_{2}, 1 \mathrm{mM}$ DTT and $2 \mathrm{mM}$ ATP at $200 \mathrm{nM}$ spastin and poly-glutamate concentrations ranging from 0 to $100 \mu \mathrm{M}$. Stock solutions $(100 \mathrm{mM})$ of poly-glutamate $(0.75-5.0 \mathrm{kDa})$ was made in water and adjusted to $\mathrm{pH}$ 7.5 with potassium hydroxide. Ten-fold serial dilutions were made in water and added to the ATPase reaction, before addition of ATP. Initial rates were calculated from the linear portion of the reaction curve. ATPase rates were corrected by subtraction of the measured release of phosphate in the absence of ATP and poly-glutamate. 


\section{Microtubule severing assays}

Microtubules were polymerized from $2 \mathrm{mg} / \mathrm{ml}$ bovine cycled brain tubulin (PurSolutions), $4 \%$ tetramethyl rodamine labeled and $1 \%$ biotin-labeled porchine brain tubulin (Cytoskeleton). Microtubules were double-cycled with the slow-hydrolyzable GTP analog guanylyl ( $\alpha, \beta)$-methylene diphosphonate (GMPCPP). The first polymerization was 1 hour and the second polymerization overnight ${ }^{81}$. The microtubules were spun down at $126,000 \mathrm{xg}$ using a TLA100 rotor (Beckman Coulter), re-suspended in warm BRB80 (80 mM K-PIPES pH 6.8, $1 \mathrm{mM} \mathrm{MgCl}_{2}, 1 \mathrm{mM}$ EGTA) and stored at $37^{\circ} \mathrm{C}$. Chambers for TIRF microscopy were assembled as described previously ${ }^{69}$. Microtubules were immobilized in the chamber using 2-mg/ml NeutrAvidin (Thermo Fisher Scientific) and imaged by TIRF microscopy in severing buffer containing BRB80, 2-mg/ml casein, $9.1 \mathrm{mM}$ 2-mercaptoethanol, 2.5\% glycerol, $50 \mathrm{mM} \mathrm{KCl}, 2.5 \mathrm{mM} \mathrm{MgCl}_{2}, 1 \mathrm{mM}$ ATP, 1\% Pluronic F127 (Life Technologies), and oxygen scavengers ${ }^{69}$. Severing reactions were carried out using $20 \mathrm{nM}$ full-length wildtype and mutants of Drosophila melanogaster spastin. Severing was scored as previously described ${ }^{20}$. Images were acquired with a Nikon Ti-E microscope equipped with a 60X 1.49 NA oil objective and a TI-TIRF adapter (Nikon) at $1 \mathrm{~Hz}$ with a $50 \mathrm{~ms}$ exposure time. Excitation was provided by a $561 \mathrm{~nm}$ laser (Agilent MLC) set to $20 \mathrm{~mW}$ before being coupled to the microscope by an optical fiber. The final pixel size for images was $90 \mathrm{~nm}$. Image acquisition was carried out using Micro-Manager ${ }^{82}$. Image analysis was carried out in Fiji. Prism (Graphpad Inc.) was used for graphing and statistical analysis.

\section{Quantification and data analysis}

$\mathrm{N}$ numbers are reported for all experiments in figure legends. Data in Fig. 4b, c were subjected to a Mann-Whitney statistical test.

\section{Homology modeling}

A human spastin hexamer homology model was generated using the online server SWISSMODEL $^{83}$ (https://swissmodel.expasy.org/). Residues 228-617 of the M1 isoform (uniprot: Q9UBP0) were used as a targeting sequence and our $3.2 \AA$ atomic model of the $D$. melanogaster spastin was used as a template.

\section{Reporting Summary}

Further information on experimental design is available in the Nature Research Reporting Summary linked to this article.

\section{Data Availability}

All data used in this study is available from the corresponding authors upon reasonable request. Electron microscopy map and the top scoring model of 5 atomic models obtained from an EM multi-model pipeline have been deposited at the Electron Microscopy Data Bank and Protein Data Bank under accession numbers EMDB: 20226 and PDB: 6P07

\section{Supplementary Material}

Refer to Web version on PubMed Central for supplementary material. 


\section{Acknowledgements}

We thank J.C. Ducom at The Scripps Research Institute High Performance Computing for computational support, and B. Anderson at The Scripps Research Institute electron microscopy facility for microscope support. We thank M. Herzik and A. Hernandes for help with atomic modeling, E. Szczesna for help with microtubule severing assays, G. Piszcek from the Biophysics Core of the National Heart, Lung and Blood Institute for help with AUC experiments, S. Chowdhury, C. Puchades, and M. Wu for helpful discussion. C.R.S. is supported by a National Science Foundation predoctoral fellowship. G.C.L. is supported as a Searle Scholar, a Pew Scholar, an Amgen Young Investigator, and by the National Institutes of Health (NIH) DP2EB020402. Computational analyses of EM data were performed using shared instrumentation funded by NIH S10OD021634 to G.C.L. A.R.M. is supported by the intramural programs of the National Institute of Neurological Disorders and Stroke (NINDS) and the National Heart, Lung and Blood Institute (NHLBI).

\section{References}

1. Mcnally FJ \& Roll-Mecak A Microtubule-severing enzymes: From cellular functions to molecular mechanism. 1-13 (2018). doi:10.1083/jcb.201612104

2. Salinas $S$ et al. Human spastin has multiple microtubule-related functions. J. Neurochem 95, $1411-$ 1420 (2005). [PubMed: 16219033]

3. Eckert T, Le DT Van, Link S, Friedmann L \& Woehlke G Spastin's Microtubule-Binding Properties and Comparison to Katanin. PLoS One 7, 1-16 (2012).

4. Wen $\mathrm{M} \&$ Wang $\mathrm{C}$ The nucleotide cycle of spastin correlates with its microtubule-binding properties. FEBS J. 280, 3868-3877 (2013). [PubMed: 23745751]

5. Evans KJ, Gomes ER, Reisenweber SM, Gundersen GG \& Lauring BP Linking axonal degeneration to microtubule remodeling by Spastin-mediated microtubule severing. J. Cell Biol 168, 599-606 (2005). [PubMed: 15716377]

6. Roll-Mecak A \& Vale RD Structural basis of microtubule severing by the hereditary spastic paraplegia protein spastin. Nature 451, 363-367 (2008). [PubMed: 18202664]

7. Roll-Mecak A \& Vale RD The Drosophila homologue of the hereditary spastic paraplegia protein, spastin, severs and disassembles microtubules. Curr. Biol 15, 650-655 (2005). [PubMed: 15823537]

8. Hazan J et al. Spastin, a new AAA protein, is altered in the most frequent form of autosomal dominant spastic paraplegia. Nat. Genet 23, 296-303 (1999). [PubMed: 10610178]

9. Blackstone C, O'Kane CJ \& Reid E Hereditary spastic paraplegias: Membrane traffic and the motor pathway. Nat. Rev. Neurosci 12, 31-42 (2011). [PubMed: 21139634]

10. Solowska JM \& Baas PW Hereditary spastic paraplegia SPG4: What is known and not known about the disease. Brain 138, 2471-2484 (2015). [PubMed: 26094131]

11. Stone MC et al. Normal Spastin Gene Dosage Is Specifically Required for Axon Regeneration. Cell Rep. 2, 1340-1350 (2012). [PubMed: 23122959]

12. Havlicek S et al. Gene dosage-dependent rescue of HSP neurite defects in SPG4 patients' neurons. Hum. Mol. Genet 23, 2527-2541 (2014). [PubMed: 24381312]

13. White SR, Evans KJ, Lary J, Cole JL \& Lauring B Recognition of C-terminal amino acids in tubulin by pore loops in Spastin is important for microtubule severing. J. Cell Biol 176, 995-1005 (2007). [PubMed: 17389232]

14. Zehr E et al. Katanin spiral and ring structures shed light on power stroke for microtubule severing. Nat. Struct. Mol. Biol 24, 717-725 (2017). [PubMed: 28783150]

15. Hartman JJ \& Vale RD Microtubule disassembly by ATP-dependent oligomerization of the AAA enzyme katanin. Science (80-. ). 286, 782-785 (1999).

16. Eckert $\mathrm{T}$ et al. Subunit Interactions and Cooperativity in the Microtubule-severing AAA ATPase Spastin. 287, 26278-26290 (2012).

17. Cummings CM, Bentley CA, Perdue SA, Baas PW \& Singer JD The Cul3/Klhdc5 E3 ligase regulates p60/katanin and is required for normal mitosis in mammalian cells. J. Biol. Chem 284, 11663-11675 (2009). [PubMed: 19261606]

18. Lu C, Srayko M \& Mains PE The Caenorhabditis elegans Microtubule-severing Complex MEI-1/ MEI-2 Katanin Interacts Differently with Two Superficially Redundant $\beta$-Tubulin Isotypes. Mol. Biol. Cell (2004). doi:10.1091/mbc.e03-06-0418 
19. Sherwood NT, Sun Q, Xue M, Zhang B \& Zinn K Drosophila spastin regulates synaptic microtubule networks and is required for normal motor function. PLoS Biol. 2, (2004).

20. Valenstein ML \& Roll-Mecak A Graded Control of Microtubule Severing by Tubulin Glutamylation. Cell 164, 911-921 (2016). [PubMed: 26875866]

21. Vemu A et al. Severing enzymes amplify microtubule arrays through lattice GTP-tubulin incorporation. Science (80-. ). 361, eaau1504 (2018).

22. Itzhak DN, Tyanova S, Cox J \& Borner GHH Global, quantitative and dynamic mapping of protein subcellular localization. Elife 5, 1-36 (2016).

23. Geimer S, Teltenkötter A, Plessmann U, Weber K \& Lechtreck KF Purification and characterization of basal apparatuses from a flagellate green alga. Cell Motil. Cytoskeleton 37, 72 85 (1997). [PubMed: 9142440]

24. Schneider A, Plessmann U, Felleisen R \& Weber K Posttranslational modifications of trichomonad tubulins; identification of multiple glutamylation sites. FEBS Lett. 429, 399-402 (1998). [PubMed: 9662457]

25. Abid Ali F et al. Cryo-EM structures of the eukaryotic replicative helicase bound to a translocation substrate. Nat. Commun 7, 10708 (2016). [PubMed: 26888060]

26. Skordalakes E \& Berger JM Structure of the Rho transcription terminator: Mechanism of mRNA recognition and helicase loading. Cell 114, 135-146 (2003). [PubMed: 12859904]

27. Taylor JL, White SR, Lauring B \& Kull FJ Crystal structure of the human spastin AAA domain. J. Struct. Biol 179, 133-137 (2012). [PubMed: 22446388]

28. Han H, Monroe N, Sundquist WI, Shen PS \& Hill CP The AAA ATPase Vps4 binds ESCRT-III substrates through a repeating array of dipeptide-binding pockets. Elife 6, 1-15 (2017).

29. Gates SN et al. Ratchet-like polypeptide translocation mechanism of the AAA+ disaggregase Hsp104. Science (80-. ). 357, 273-279 (2017).

30. De la Peña AH, Goodall EA, Gates SN, Lander GC \& Martin A Substrate-engaged 26S proteasome structures reveal mechanisms for ATP-hydrolysis-driven translocation. Science (80-. ). 362, (2018).

31. Augustyniak R \& Kay LE Cotranslocational processing of the protein substrate calmodulin by an AAA+ unfoldase occurs via unfolding and refolding intermediates. Proc. Natl. Acad. Sci 115, E4786-E4795 (2018). [PubMed: 29735657]

32. Schlieker C et al. Substrate recognition by the AAA+ chaperone ClpB. Nat. Struct. Mol. Biol. 11, 607-615 (2004). [PubMed: 15208691]

33. Puchades $\mathrm{C}$ et al. Structure of the mitochondrial inner membrane AAA+ protease YME1 gives insight into substrate processing. Science (80-. ). 358, eaao0464 (2017).

34. Ripstein ZA, Huang R, Augustyniak R, Kay LE \& Rubinstein JL Structure of a AAA+ unfoldase in the process of unfolding substrate. Elife 6, 1-14 (2017).

35. Alfieri C, Chang L \& Barford D Mechanism for remodelling of the cell cycle checkpoint protein MAD2 by the ATPase TRIP13. Nature 559, 274-278 (2018). [PubMed: 29973720]

36. Scott A et al. Structural and mechanistic studies of VPS4 proteins. EMBO J. 24, 3658-3669 (2005). [PubMed: 16193069]

37. Roll-Mecak A Intrinsically disordered tubulin tails: Complex tuners of microtubule functions? Semin. Cell Dev. Biol 37, 11-19 (2015). [PubMed: 25307498]

38. Hinnerwisch J, Fenton WA, Furtak KJ, Farr GW \& Horwich AL Loops in the central channel of ClpA chaperone mediate protein binding, unfolding, and translocation. Cell 121, 1029-1041 (2005). [PubMed: 15989953]

39. Lee J et al. Structural determinants for protein unfolding and translocation by the Hsp104 protein disaggregase. Biosci. Rep 37, BSR20171399 (2017). [PubMed: 29175998]

40. Charvin D et al. Mutations of SPG4 are responsible for a loss of function of spastin, an abundant neuronal protein localized in the nucleus. Hum. Mol. Genet 12, 71-78 (2003). [PubMed: 12490534]

41. Mészárosová AU et al. SPAST mutation spectrum and familial occurrence among Czech patients with pure hereditary spastic paraplegia. J. Hum. Genet 61, 845-850 (2016). [PubMed: 27334366] 
42. Ishiura $\mathrm{H}$ et al. Molecular epidemiology and clinical spectrum of hereditary spastic paraplegia in the Japanese population based on comprehensive mutational analyses. J. Hum. Genet 59, 163-172 (2014). [PubMed: 24451228]

43. Depienne $\mathrm{C}$ et al. Spastin mutations are frequent in sporadic spastic paraparesis and their spectrum is different from that observed in familial cases. J. Med. Genet 43, 259-265 (2006). [PubMed: 16055926]

44. Tang BS et al. Clinical features of hereditary spastic paraplegia with thin corpus callosum: Report of 5 Chinese cases. Chin. Med. J. (Engl) (2004).

45. Hentati A et al. Novel mutations in spastin gene and absence of correlation with age at onset of symptoms. Neurology 55, 1388-90 (2000). [PubMed: 11087788]

46. Meijer IA, Hand CK, Cossette P, Figlewicz DA \& Rouleau GA Spectrum of SPG4 mutations in a large collection of North American families with hereditary spastic paraplegia. Arch. Neurol 281286 (2002). doi:10.1001/archneur.59.2.281 [PubMed: 11843700]

47. Patrono C et al. Autosomal dominant hereditary spastic paraplegia: DHPLC-based mutation analysis of SPG4 reveals eleven novel mutations. Hum. Mutat 25, 506 (2005).

48. Balicza P et al. Genetic background of the hereditary spastic paraplegia phenotypes in Hungary An analysis of 58 probands. J. Neurol. Sci. 364, 116-121 (2016). [PubMed: 27084228]

49. Bürger J et al. Hereditary spastic paraplegia caused by mutations in the SPG4 gene. Eur. J. Hum. Genet (2000). doi:10.1038/sj.ejhg.5200528

50. Elert-Dobkowska E et al. Molecular spectrum of the SPAST, ATL1 and REEP1 gene mutations associated with the most common hereditary spastic paraplegias in a group of Polish patients. J. Neurol. Sci 359, 35-39 (2015). [PubMed: 26671083]

51. Lu X et al. Genetic analysis of SPG4 and SPG3A genes in a cohort of Chinese patients with hereditary spastic paraplegia. J. Neurol. Sci 347, 368-371 (2014). [PubMed: 25454648]

52. Park H et al. Mutational spectrum of the SPAST and ATL1 genes in Korean patients with hereditary spastic paraplegia. J. Neurol. Sci 357, 167-172 (2015). [PubMed: 26208798]

53. Polymeris AA et al. A series of Greek children with pure hereditary spastic paraplegia: clinical features and genetic findings. J. Neurol 263, 1604-1611 (2016). [PubMed: 27260292]

54. Aulitzky A et al. A complex form of hereditary spastic paraplegia in three siblings due to somatic mosaicism for a novel SPAST mutation in the mother. J. Neurol. Sci 347, 352-355 (2014). [PubMed: 25315759]

55. McDermott CJ et al. Clinical features of hereditary spastic paraplegia due to spastin mutation. Neurology (2006). doi:10.1212/01.wnl.0000223315.62404.00

56. Yabe I, Sasaki H \& Tashiro K Spastin gene mutation in Japanese with hereditary spastic paraplegia. J. Med ... 39, 14-15 (2002).

57. Dong EL et al. Clinical spectrum and genetic landscape for hereditary spastic paraplegias in China. Mol. Neurodegener 13, 1-14 (2018). [PubMed: 29310663]

58. Luo Y et al. A diagnostic gene chip for hereditary spastic paraplegias. Brain Res. Bull 97, 112-118 (2013). [PubMed: 23850684]

59. Shoukier $\mathrm{M}$ et al. Expansion of mutation spectrum, determination of mutation cluster regions and predictive structural classification of SPAST mutations in hereditary spastic paraplegia. Eur. J. Hum. Genet 17, 187-194 (2009). [PubMed: 18701882]

60. Fonknechten N et al. Spectrum of SPG4 mutations in autosomal dominant spastic paraplegia. Hum. Mol. Genet. 9, 637-644 (2000). [PubMed: 10699187]

61. Proukakis C, Moore D, Labrum R, Wood NW \& Houlden H Detection of novel mutations and review of published data suggests that hereditary spastic paraplegia caused by spastin (SPAST) mutations is found more often in males. J. Neurol. Sci 306, 62-65 (2011). [PubMed: 21546041]

62. Solowska JM et al. Pathogenic Mutation of Spastin Has Gain-of-Function Effects on Microtubule Dynamics. J. Neurosci 34, 1856-1867 (2014). [PubMed: 24478365]

63. Qiang L et al. Hereditary spastic paraplegia: gain-of-function mechanisms revealed by new transgenic mouse. Hum. Mol. Genet 28, 1136-1152 (2018). 
64. Errico A, Ballabio A \& Rugarli EI Spastin, the protein mutated in autosomal dominant hereditary spastic paraplegia, is involved in microtubule dynamics. Hum. Mol. Genet 11, 153-163 (2002). [PubMed: 11809724]

65. Crippa F et al. Eight novel mutations in SPG4 in a large sample of patients with hereditary spastic paraplegia. Arch. Neurol. (2006). doi:10.1001/archneur.63.5.750

66. França MC et al. SPG4-related hereditary spastic paraplegia: Frequency and mutation spectrum in Brazil. Clin. Genet. 86, 194-196 (2014). [PubMed: 24033003]

67. Kim T-H et al. Mutation analysis of SPAST, ATL1, and REEP1 in Korean Patients with Hereditary Spastic Paraplegia. J. Clin. Neurol 10, 257-61 (2014). [PubMed: 25045380]

68. Gillespie MK, Humphreys P, McMillan HJ \& Boycott KM Association of Early-Onset Spasticity and Risk for Cognitive Impairment With Mutations at Amino Acid 499 in SPAST. J. Child Neurol (2018). doi:10.1177/0883073818756680

\section{Methods References}

69. Ziolkowska N \& Roll-Mecak A In Vitro Microtubule Severing Assays. Neurochem. Int 37, 399 400 (2013).

70. Schuck P Size-distribution analysis of macromolecules by sedimentation velocity ultracentrifugation and Lamm equation modeling. Biophys. J 78, 1606-1619 (2000). [PubMed: 10692345]

71. Brown PH, Balbo A \& Schuck P Using prior knowlede in the determination of macromolecular size-disrtributions by analytical ultracentrifugation. Biomacromolecules 8, 2011-2024 (2007). [PubMed: 17521163]

72. Carragher B et al. Leginon: an automated system for acquisition of images from vitreous ice specimens. J. Struct. Biol 132, 33-45 (2000). [PubMed: 11121305]

73. Voss NR, Yoshioka CK, Radermacher M, Potter CS \& Carragher B DoG Picker and TiltPicker: Software tools to facilitate particle selection in single particle electron microscopy. J. Struct. Biol 166, 205-213 (2009). [PubMed: 19374019]

74. Punjani A, Rubinstein JL, Fleet DJ \& Brubaker MA CryoSPARC: Algorithms for rapid unsupervised cryo-EM structure determination. Nat. Methods 14, 290-296 (2017). [PubMed: 28165473]

75. Scheres SHW RELION: Implementation of a Bayesian approach to cryo-EM structure determination. J. Struct. Biol 180, 519-530 (2012). [PubMed: 23000701]

76. Pettersen EF et al. UCSF Chimera - A visualization system for exploratory research and analysis. J. Comput. Chem 25, 1605-1612 (2004). [PubMed: 15264254]

77. Adams PD et al. PHENIX: A comprehensive Python-based system for macromolecular structure solution. Acta Crystallogr. Sect. D Biol. Crystallogr (2010). doi:10.1107/S0907444909052925

78. Emsley P \& Cowtan K Coot: Model-building tools for molecular graphics. Acta Crystallogr. Sect. D Biol. Crystallogr (2004). doi:10.1107/S0907444904019158

79. Herzik MA, Fraser JS \& Lander GC A Multi-model Approach to Assessing Local and Global Cryo-EM Map Quality. Structure (2018). doi:10.1016/j.str.2018.10.003

80. Chen VB et al. MolProbity: All-atom structure validation for macromolecular crystallography. Acta Crystallogr. Sect. D Biol. Crystallogr (2010). doi:10.1107/S0907444909042073

81. Gell C et al. Purification of tubulin from porcine brain. Methods Mol. Biol (2011). doi: 10.1007/978-1-61779-252-6_2

82. Edelstein $\mathrm{AD}$ et al. Advance methods of microscope control using microManager software. 1, 1-18 (2015).

83. Waterhouse A et al. SWISS-MODEL: Homology modelling of protein structures and complexes. Nucleic Acids Res. 46, W296-W303 (2018). [PubMed: 29788355]

84. Barad BA et al. EMRinger: Side chain-directed model and map validation for 3D cryo-electron microscopy. Nat. Methods 12, 943-946 (2015). [PubMed: 26280328]

85. Cardone G, Heymann JB \& Steven AC One number does not fit all: Mapping local variations in resolution in cryo-EM reconstructions. J. Struct. Biol 184, 226-236 (2013). [PubMed: 23954653] 
86. Meng EC, Pettersen EF, Couch GS, Huang CC \& Ferrin TE Tools for integrated sequence-structure analysis with UCSF Chimera. BMC Bioinformatics 7, 1-10 (2006). [PubMed: 16393334] 

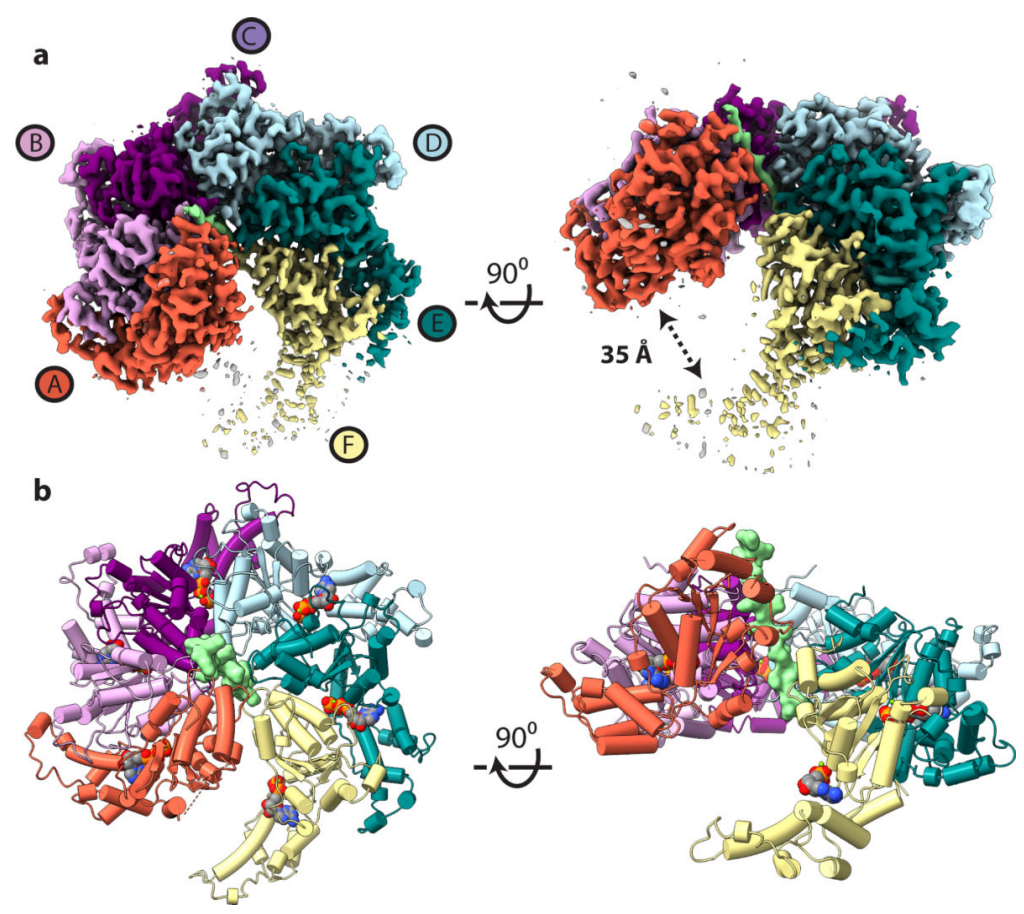

Figure 1. The architecture of the substrate-bound spastin hexamer in a split lock-washer conformation.

(a) Top (Left) and side view (Right) of the EM map of the spastin hexamer with individual protomers colored separately. Substrate density is colored in light green. The $\sim 35 \AA$ gap between protomer A and F is apparent in the sideview. (b) Top (Left) and side view (Right) of the atomic model in ribbon representation. Nucleotides are depicted as space-filling models and the polyglutamate peptide substrate is shown in surface representation. 


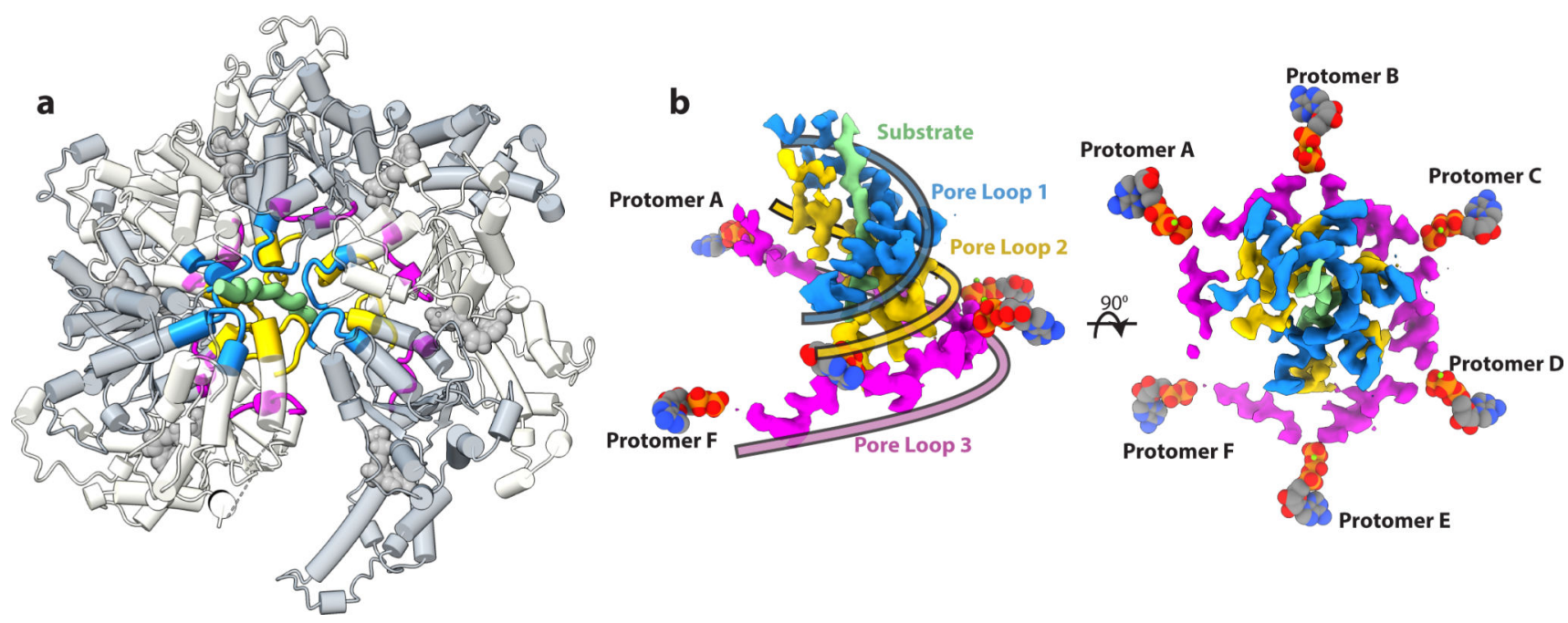

Figure 2. A double-helical staircase of pore loops surrounds the substrate and is coupled by a third pore loop spiral to the ATP binding site

(a) The three solvent exposed pore loops highlighted on the spastin hexamer structure. Pore loop 1 is colored in blue, pore loop 2 in gold, and pore loop 3 in magenta. (b) Side (Left) and top (Right) view of the EM density of the triple spiral staircase generated by pore loops 1, 2 and 3. Pore loops 1 and pore loops 2 form a tight double spiral that engages to the peptide substrate shown in light green. Pore loops 3 form a shallow spiral between pore loops 1-2 and the nucleotide. Ribbons colored as their corresponding pore loops are overlaid on the EM map to highlight the trajectory of the three pore loop spirals. 
a

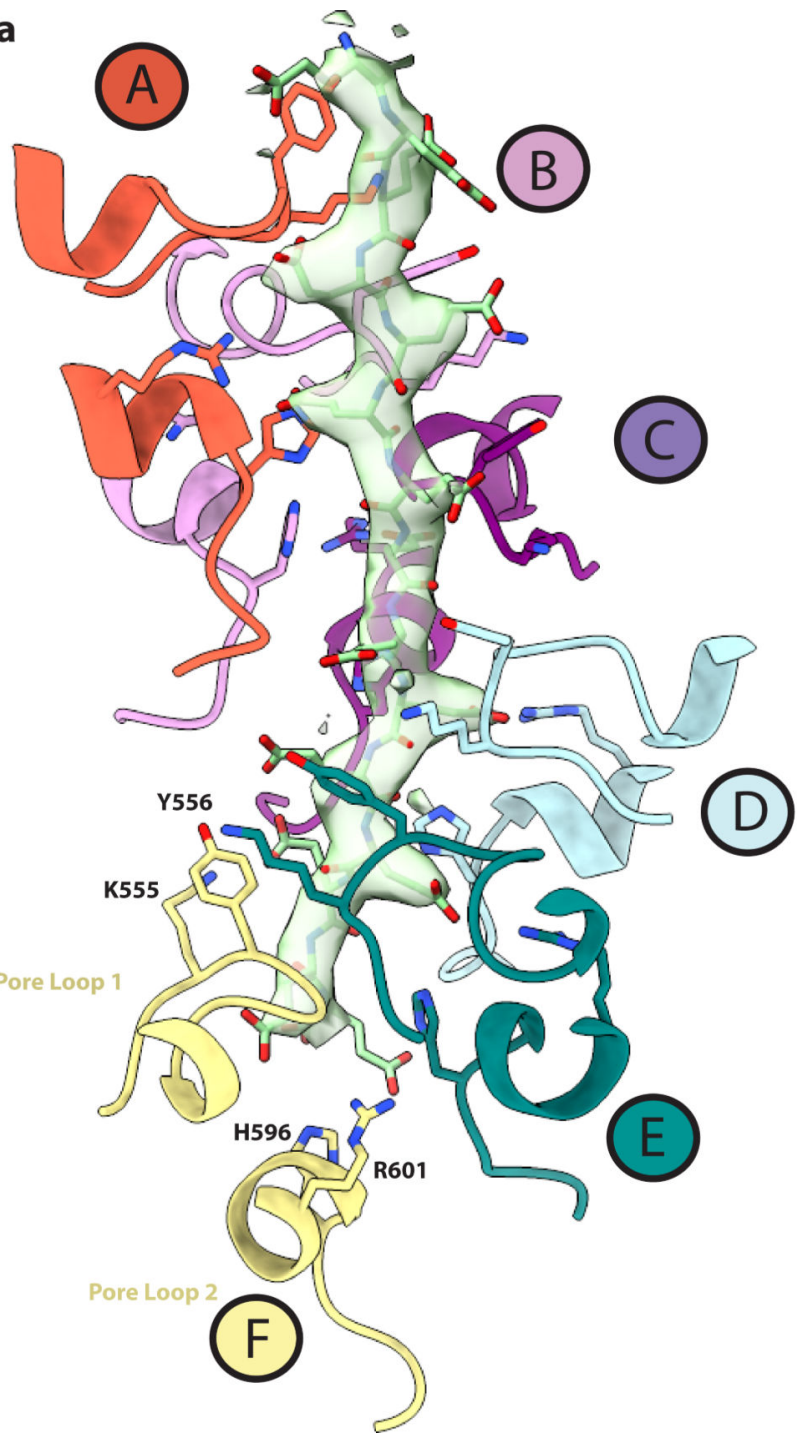

b

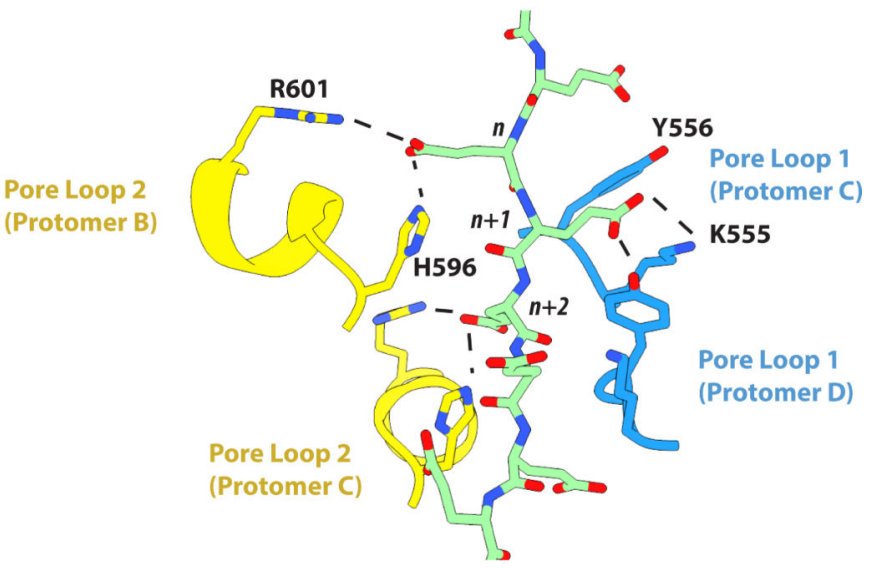

C

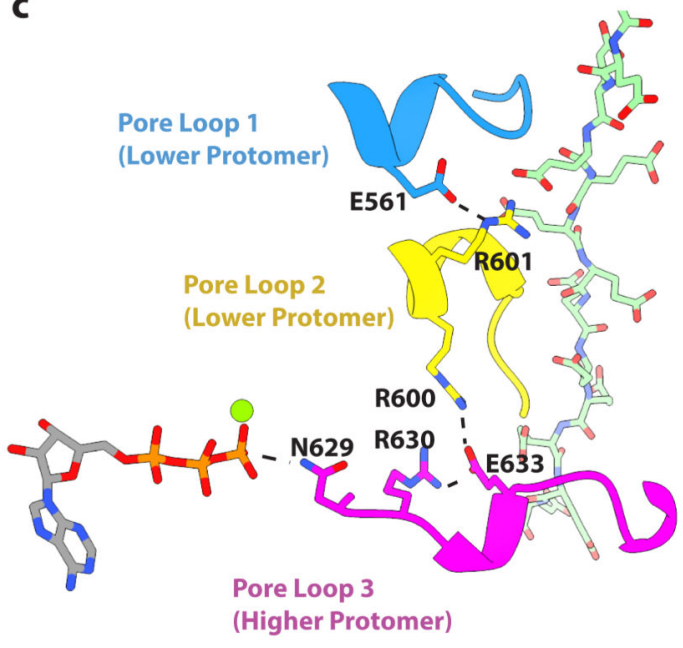

Figure 3. A pore loop network couples nucleotide binding to substrate engagement and oligomerization.

(a) Pore loops 1 and 2 interacting with the polyglutamate substrate. Substrate density is shown as a transparent surface with the polyglutamate model in light green. Pore loops are colored according to their assigned protomer, as in Figure 1. Residues that interact directly with the substrate (K555, Y556, H596, R601) are shown in stick representation. (b) Interactions between the substrate and conserved residues in pore loops 1 and 2. (c) A network of interactions between charged residues connects the peptide substrate to the nucleotide. Hydrogen bonds are shown as dashed lines. All interactions depicted have a measured distance of less than $4 \AA$. 
a
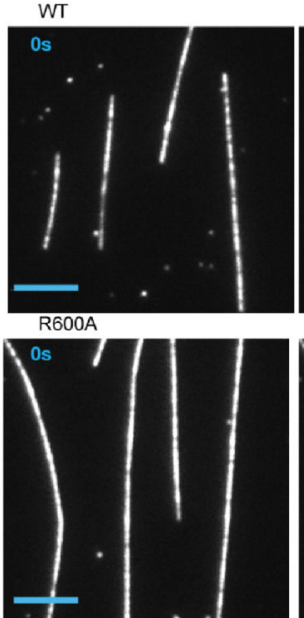
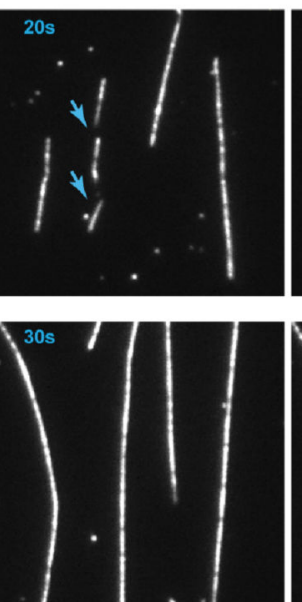
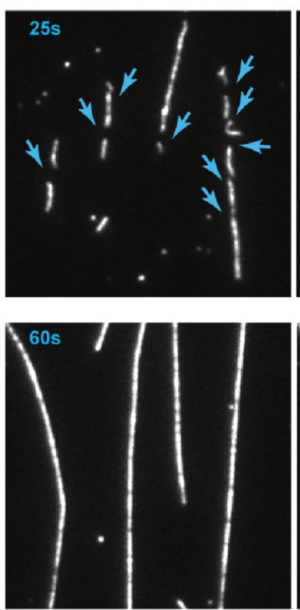
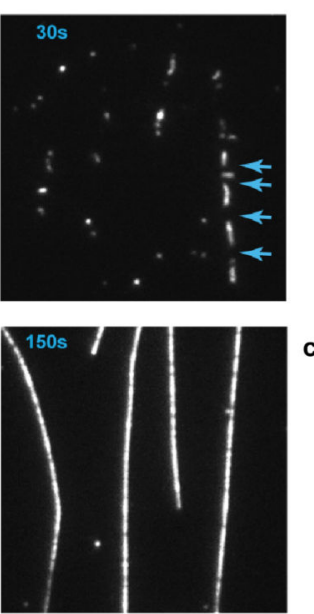

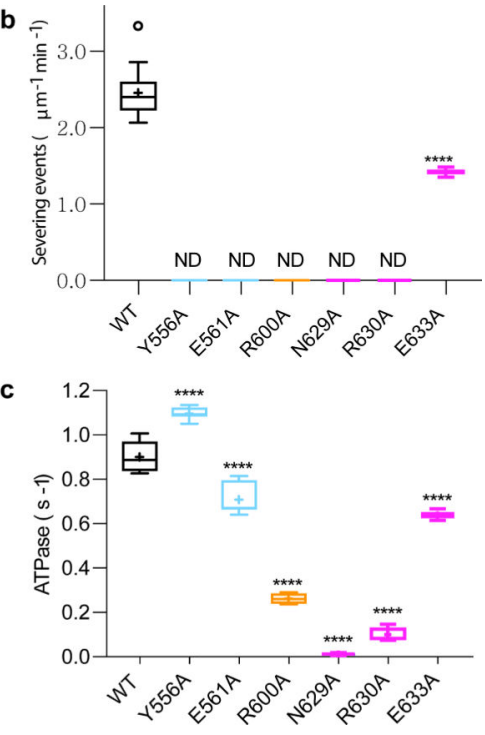

Figure 4. Mutations in the three pore loops impair ATPase and microtubule severing.

(a) Microtubule severing assays of wild-type spastin and pore loop mutant R600A. Severing events are highlighted with blue arrows. Scale bar, $5 \mu \mathrm{m}$. (b) Tukey plots of severing rates of structure-based spastin mutants, $\mathrm{n}=30$ microtubules from 2, 4, 4, 4, 4, 4 and 3 chambers for wild-type, Y556A, E561A, R600A, E629A, R630A, E633A mutants (c) and ATPase rates of structure-based spastin mutants, $\mathrm{n}=24,16,12,8,8,7,8$ for wild-type, Y556A, E561A, R600A, E629A, R630A, E633A mutants. Plus denotes mean, outliers shown as circles; ****, $\mathrm{p}<0.0001$, determined by the Mann-Whitney test. ND, not detectable. Pore loop 1, 2 and 3 mutants shown in cyan, orange and magenta, respectively. 
a

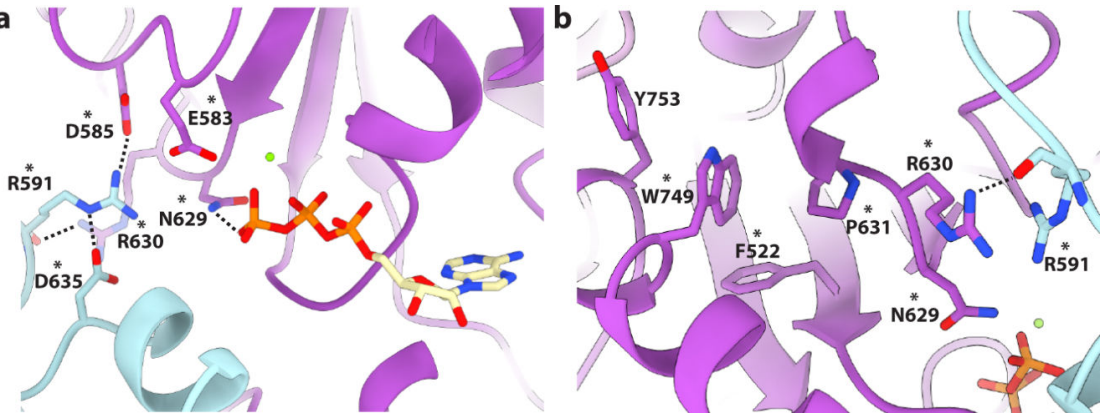

Figure 5. An allosteric network mutated in HSP couples substrate binding to oligomerization and ATP hydrolysis

(a) Interaction network centered on R591. Residues colored plum and cyan for protomers B and $\mathrm{C}$, respectively. Residues mutated in HSP are indicated with an asterix. All interactions depicted have a measured distance of less than $4 \AA$. (b) W749 makes van der Walls contacts with several hydrophobic residues mutated in HSP and connects pore loop 3 to the Cterminal helix a 11 involved in oligomerization. Residues are colored purple and cyan for protomers C and D, respectively. Residues mutated in HSP are indicated with an asterix. All interactions depicted have a measured distance of less than $4 \AA$. 
a

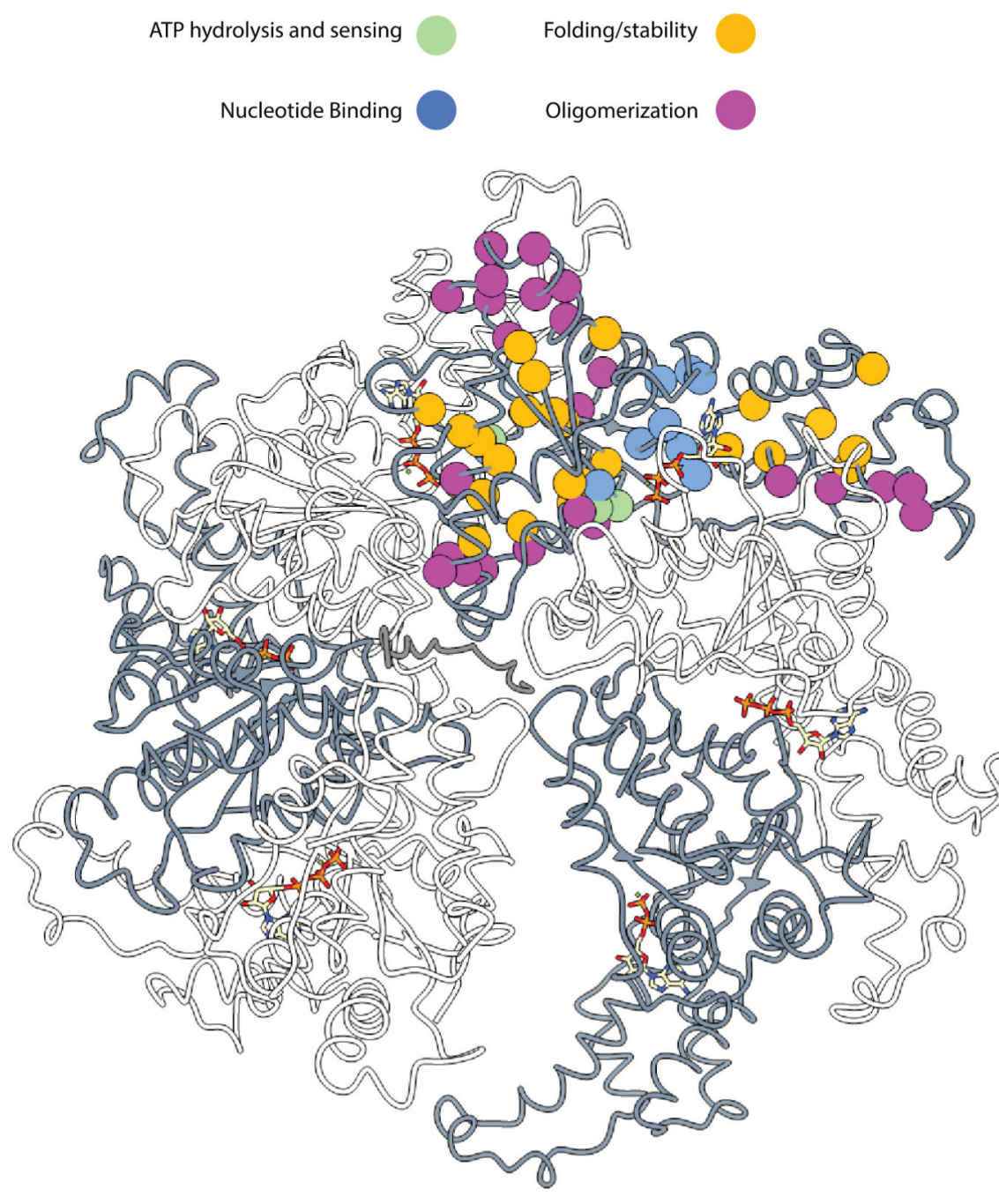

b

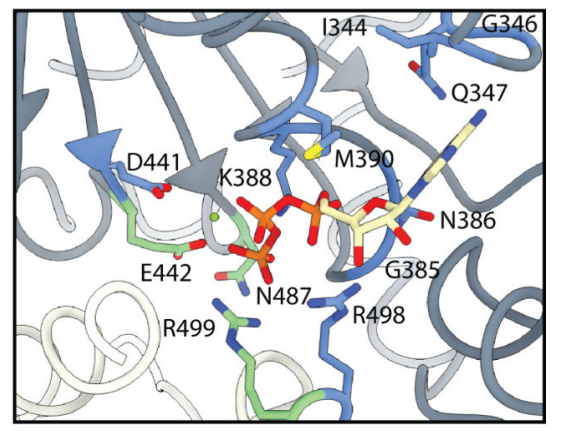

C

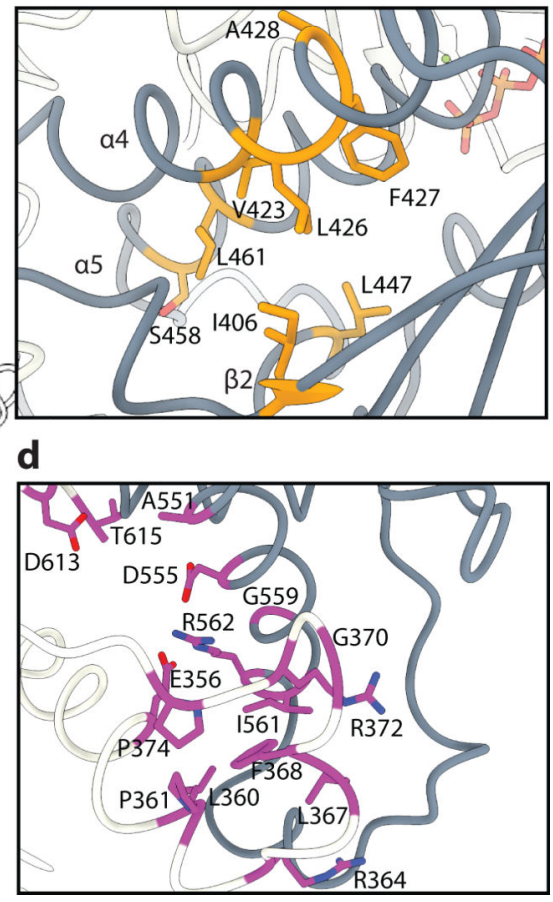

Figure 6. Structure-based insight into the mechanism of action of human spastin HSP disease mutations.

(a) Homology model of the human spastin hexamer depicting the location of missense mutations identified in HSP. Protomers are colored in alternating grey and white around the hexamer. Mutations are depicted as spheres at their $\mathrm{C}_{\mathrm{a}}$ position and colored according to their proposed mechanism of action: interference with nucleotide binding (blue), defects in ATP hydrolysis and $\gamma$-phosphate sensing (green), disruption of interactions important for folding or conferring stability to the protomer (orange), and disruption of oligomerization interfaces (magenta). (b) Close-up view of the nucleotide pocket, showing a large number of mutations that likely disrupt nucleotide binding or impair ATP hydrolysis and sensing. (c) Close-up view of hydrophobic interfaces between $a 4$, a 5 and $\beta 2$ highlighting potentially destabilizing HSP mutations. (d) Close-up view of the NBD-HBD interaction interface between protomers showing a high-density of HSP mutations. 
Table 1.

Cryo-EM data collection, refinement and validation statistics

\begin{tabular}{|c|c|}
\hline & Spastin $^{\mathrm{E} 583 Q}$ (PDB 6P07) (EMDB 20226) \\
\hline \multicolumn{2}{|l|}{ Data collection and Processing } \\
\hline Microscope & Thermo Fischer Talos Arctica \\
\hline Camera & Gatan K2 Summit DED \\
\hline Magnification (nominal) & $36,000 x$ \\
\hline Magnification (calibrated) & $43478.3 x$ \\
\hline Voltage (kV) & 200 \\
\hline Total electron exposure $\left(\mathrm{e}^{-} / \AA^{2}\right)$ & 52 \\
\hline Exposure rate (e-/pixel/sec) & 5.6 \\
\hline Defocus range $(\mu \mathrm{m})$ & -1.0 to -2.0 \\
\hline Pixel size $(\AA)$ & 1.15 \\
\hline Micrographs collected (no.) & 2,534 \\
\hline Micrographs used (no.) & 2,534 \\
\hline Total extracted particles (no.) & $2,736,865$ \\
\hline Refined particles (no.) & $1,259,553$ \\
\hline Final particles (no.) & 488,385 \\
\hline Symmetry imposed & $\mathrm{C} 1$ \\
\hline \multicolumn{2}{|l|}{ Resolution (global) } \\
\hline FSC 0.5 (unmasked/masked) & $7.0 / 3.6$ \\
\hline FSC 0.143 (unmasked/masked) & $4.3 / 3.2$ \\
\hline Resolution Range (local) & $3-5$ \\
\hline \multicolumn{2}{|l|}{ Model composition } \\
\hline Nonhydrogen atoms & 14,089 \\
\hline Protein residues & 1804 \\
\hline Ligands & 12 \\
\hline \multicolumn{2}{|l|}{ Refinement } \\
\hline Initial model used (PDB code) & 3B9P \\
\hline Average FSC & 3.2 \\
\hline \multicolumn{2}{|l|}{$B$ factors $\left(\AA^{2}\right)$} \\
\hline Protein residues & 46.8 \\
\hline Ligands & 50.5 \\
\hline \multicolumn{2}{|l|}{ R.m.s. deviations } \\
\hline Bond lengths $(\AA)$ & 0.01 \\
\hline Bond angles $\left(^{\circ}\right)$ & 1.14 \\
\hline \multicolumn{2}{|l|}{ Validation } \\
\hline MolProbity score & 1.46 \\
\hline Clashscore & 3.05 \\
\hline Poor rotamers $(\%)$ & 0.0 \\
\hline C-beta deviations & 0 \\
\hline Mean per-residue Ca RMSD $(\AA)$ & 0.64 \\
\hline
\end{tabular}




\begin{tabular}{|c|c|}
\hline & Spastin $^{E 583 Q}$ (PDB 6P07) (EMDB 20226) \\
\hline Per-residue Ca RMSD range $(\AA)$ & $0.03-5.66$ \\
\hline \multicolumn{2}{|l|}{ Ramachandran plot } \\
\hline Favored $(\%)$ & $94.65 \%$ \\
\hline Allowed (\%) & $5.35 \%$ \\
\hline Disallowed (\%) & $0.00 \%$ \\
\hline EMRinger score ${ }^{84}$ & 3.00 \\
\hline CaBLAM outliers & $3.35 \%$ \\
\hline
\end{tabular}

amachandran plot

Favored (\%)

$94.65 \%$

3

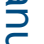

ค

후

疍

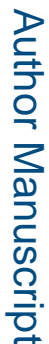

Nat Struct Mol Biol. Author manuscript; available in PMC 2020 January 08. 